\title{
Transmission electron microscopy study on pre-strained InGaN/GaN quantum wells
}

\author{
Yung-Sheng Chen, Li-Chieh Yao, Yu-Li Lin, Ling Hung, Chi-Feng Huang, Tsung-Yi Tang, \\ Jeng-Jie Huang, Wen-Yu Shiao, C.C. Yang* \\ Department of Electrical Engineering, Graduate Institute of Electro-Optical Engineering, National Taiwan University, 1, Roosevelt Road, Section 4, \\ Taipei, Taiwan, $R O C$
}

Received 19 June 2006; received in revised form 31 July 2006; accepted 30 August 2006

Communicated by R. Fornari

Available online 10 October 2006

\begin{abstract}
We use the techniques of high-resolution transmission electron microscopy and strain state analysis (SSA) to show the material nanostructures of two InGaN/GaN quantum-well (QW) samples. In one of the samples, a low-indium InGaN/GaN QW is grown before five high-indium ones, which are grown under the same conditions as those for growing the five QWs in another sample (the control sample). From the calibrations of the average indium contents of those QWs based on the SSA images, it is found that the QWs close to the low-indium one have higher indium contents than those in the control sample. Such an increase of indium incorporation is attributed to the pre-strain effect of the low-indium QW on the barrier layer right above it. The pre-strain effect diminishes along the growth of more QWs. This effect represents an effective approach for increasing indium contents for implementing yellow-red light-emitting diodes based on InGaN/GaN QW structures.
\end{abstract}

(C) 2006 Published by Elsevier B.V.

PACS: 73.21

Keywords: A3. Metalorganic vapor phase; A3. Quantum wells; B1. Nitrides; B2. Semiconducting III-V materials

\section{Introduction}

Because of the large lattice mismatch between $\mathrm{GaN}$ and $\mathrm{InN}$, strains exist at the junctions of $\mathrm{InGaN}$ and $\mathrm{GaN}$ layers such as that in an $\mathrm{InGaN} / \mathrm{GaN}$ quantum-well (QW) structure. Such a strain distribution is strongly related to the occurrence of the spinodal decomposition process in such a QW, which may lead to strong composition fluctuation and the formation of indium-rich nano-clusters [1-3]. With the occurrence of the spinodal decomposition, such a hetero-structure strain is more or less relaxed [4]. On the other hand, without the spinodal decomposition, the preserved strain leads to the quantum-confined Stark effect (QCSE) [5]. In this situation, the well (barrier) layer experiences compressive (tensile) strain. The strain condi-

\footnotetext{
${ }^{*}$ Corresponding author. Tel.: + 886223657624 ; fax: +886223652637.

E-mail address: ccy@cc.ee.ntu.edu.tw (C.C. Yang).
}

tions in the barriers of an $\mathrm{InGaN} / \mathrm{GaN}$ multiple $\mathrm{QW}$ structure build the connections of the growth and emission properties between neighboring wells. In other words, if a barrier is strained in a tensile manner, the incorporation of indium in the next QW layer becomes more effective leading to a higher indium content and hence generally a longer emission wavelength [6,7]. Therefore, the control of strain distribution is a key to the growth of high-indium InGaN compound.

The growth of a high-indium InGaN/GaN QW structure is important for developing full-color display and solidstate lighting. For full-color display, particularly for microdisplay, blue, green, and red emissions from a single-chip are desirable. For white-light generation in the application of solid-state lighting, the growth of InGaN/GaN QWs for efficient orange-red emission implies the feasibility of fabricating single-chip all-semiconductor white-light LEDs by stacking different QWs for color mixing [8-11]. Such a 
phosphor-free LED will have the advantages of energy saving, higher reliability, and possibly lower cost. So far, the only available semiconductor compound for covering the whole visible range is InGaN. For green emission from an $\mathrm{InGaN} / \mathrm{GaN}$ QW structure, normally the required indium content is around 15\%. To push even longer wavelength, the growth of $\mathrm{InGaN} / \mathrm{GaN} \mathrm{QWs}$ of even higher indium contents becomes generally difficult.

In this paper, we use the technique of transmission electron microscopy (TEM) for demonstrating the prestrain effect on the growth of InGaN/GaN multiple QWs. In particular, we use the strain state analysis (SSA) method, based on the atomic-scale TEM images, for showing the compositional fluctuations and calibrating the average indium contents of those QWs. The pre-strain effect is implemented by growing a low-indium InGaN/ GaN QW before the growth of high-indium QWs. It is found that the pre-strain effect leads to the increase of indium incorporation and hence the emission of longer wavelengths. The increase of indium incorporation is particularly significant in those high-indium QWs close to the low-indium one. The increase of indium incorporation under the pre-strain effect is identified by comparing the results with those of a control sample, in which five QWs are deposited under the same growth conditions as those for the high-indium QWs of the pre-strained sample.

This paper is organized as follows: In Section 2, the conditions for sample growth and TEM measurement are described. The TEM and the SSA images are shown in Section 3. Then, basic optical characterization results are presented in Section 4. Discussions about the material analysis results are given in Section 5. Finally, conclusions are drawn in Section 6.

\section{Sample preparation and experimental procedures}

To observe the pre-strain effect, two InGaN/GaN QW samples were grown with metal-organic chemical vapor deposition (MOCVD) for comparison. In sample A (the control sample), after a 2- $\mu \mathrm{m}$ n-type GaN layer (grown at $1070{ }^{\circ} \mathrm{C}$ ), five periods of $\mathrm{InGaN} / \mathrm{GaN}$ QW, with $3 \mathrm{~nm}$ in the well thickness (grown at $680^{\circ} \mathrm{C}$ ) and $16 \mathrm{~nm}$ in the barrier thickness, were deposited. In the growth of such a barrier layer (except the barrier before the first QW), after the growth of a $\sim 2-\mathrm{nm} \mathrm{GaN}$ cap layer at the same temperature as that for the well $\left(680^{\circ} \mathrm{C}\right)$, the growth was interrupted. During the interruption, the wafer temperature was ramped to $800{ }^{\circ} \mathrm{C}$ and $\mathrm{H}_{2}$ of $500 \mathrm{sccm}$ was added to the growth chamber. After the interruption, a 14-nm GaN layer was grown to form the $16-\mathrm{nm}$ barrier. The GaN cap layer between the InGaN well and the higher-temperature GaN barrier meant to protect the InGaN QW from indium desorption during the temperature ramping and the $\mathrm{H}_{2}$ addition [12]. After the growth of the five QWs, a $20-\mathrm{nm}$ $\mathrm{p}-\mathrm{Al}_{0.2} \mathrm{Ga}_{0.8} \mathrm{~N}$ layer, followed by a $120-\mathrm{nm} \mathrm{p}-\mathrm{GaN}$ layer (both grown at $930^{\circ} \mathrm{C}$ ), was grown. In the pre-strained sample (sample B), the epi-structure is the same as that of sample A except that an extra $\mathrm{InGaN} / \mathrm{GaN}$ QW of a lower indium content (about $7 \%$, grown at $745^{\circ} \mathrm{C}$ ) was inserted between the $\mathrm{n}-\mathrm{GaN}$ layer and the five high-indium QWs. No growth interruption procedures were used for the barriers right below and above the extra QW. They were grown at the same temperature as that for this well $\left(745^{\circ} \mathrm{C}\right)$. This growth procedure of no interruption was also applied to the barrier layer before the first QW in sample A.

We first characterize the two samples with the X-ray diffraction (XRD) measurement along the $\left(\begin{array}{llll}0 & 0 & 0 & 2\end{array}\right)$ direction using a Bede D1 system. Fig. 1 shows the XRD patterns of the two samples. Here, the sharp peaks at the origin correspond to the contributions of GaN. For sample $\mathrm{A}$, the clear second- and third-order maxima indicate the high QW quality of this sample. The average indium content in the five QWs was estimated to be $14 \%$. For sample $\mathrm{B}$, although there are five QWs grown under the same condition as that for sample A, the distribution of the secondary maxima in its XRD pattern is quite different from that of sample A. The dashed curve in Fig. 1 clearly indicates that the QW periodicity in sample B is weaker. This result implies that the five QWs designated for green emission in sample B are not uniform, either in average indium content or in well thickness, although they were grown under the same condition.

The TEM investigations were performed using a Philips Tecnai F30 field emission electron microscope using an accelerating voltage of $300 \mathrm{kV}$ and a probe forming lens of $C_{\mathrm{s}}=1.2 \mathrm{~mm}$. Those high-resolution micrographs for SSA were taken with two-electron-beam interference. The current density focused onto the samples was estimated to be lower than $16 \mathrm{~A} / \mathrm{cm}^{2}$. With the two-beam interference data, we could perform the SSA for composition distribution images $[13,14]$. In the TEM operation, to avoid the possible influence of electron illumination on the recorded results, the images were always taken within 5-10 s after the electron beam was focused onto the target spots. Our experiments showed that the images were not significantly

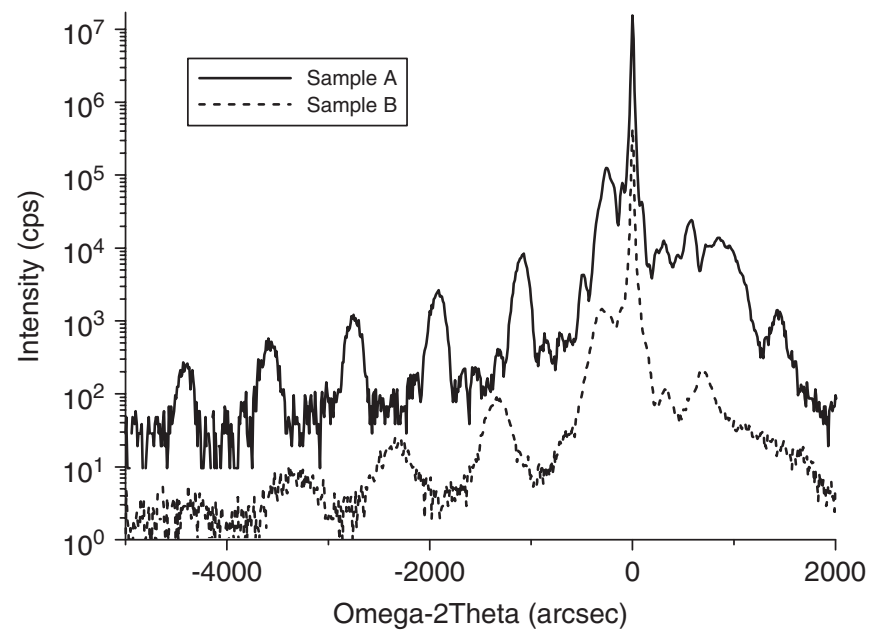

Fig. 1. XRD patterns of the two samples. 
changed within $30 \mathrm{~s}$ of the illumination of focused electron beams. To obtain the SSA images, the [ $\left[\begin{array}{llll}0 & 0 & 0 & 1\end{array}\right]$ lattice size distributions were first calibrated from the lattice fringe images. The lattice size distribution is represented by a parameter, $d$, defined as the ratio of the local lattice size over that of GaN. Then, the indium composition distribution can be calibrated based on Vegard's law subject to a control factor. This factor is related to the TEM sample thickness [13,14]. However, it is usually difficult to determine this thickness. Because the boundaries of the low-indium QW in sample $\mathrm{B}$ is quite clear and its composition is quite uniform (see Fig. 7(a)), we can use its indium content as the reference for calibrating those of other QWs based on the variation of the parameter $d$. From our previous study of MOCVD growth, the indium content of this low-indium QW is calibrated to be about $7 \%$. This indium content corresponds to the value of $d$ at 1.033. Based on this correspondence, we can estimate the thickness of our TEM samples as around $50 \mathrm{~nm}$, which is quite reasonable. Because $(d-1)$ is linearly proportional to the indium composition percentage, we can have

$\frac{7 \%}{(1.033-1)}=\frac{x}{(d-1)}$

Here, $x$ is the average indium composition percentage of a QW. Therefore, the local indium composition can be obtained by using the formula of $212 \% x(d-1)$. In optical characterization, the photoluminescence (PL) measurements were carried out with the $325-\mathrm{nm}$ line of a $35 \mathrm{~mW}$ $\mathrm{He}-\mathrm{Cd}$ laser as the excitation source. The samples were placed in a cryostat for temperature-dependent measurements ranging from 10 to $300 \mathrm{~K}$.

\section{TEM and SSA images}

Fig. 2 shows a TEM image of sample A. Here, five equally spaced QWs can be clearly seen as indicated with the white arrows. Also, the p-AlGaN and p-GaN layers grown on top of the five QWs can be identified. Then, in Fig. 3, we show a TEM image of higher magnification to demonstrate more clearly the nanostructures of the first three QWs (from the top). Here, although the QW shapes can be clearly observed, nano-scale clusters can be identified. The general structures of the three QWs are the same. Fig. 4 shows a typical SSA image of a QW in sample A. This particular image demonstrates the composition mapping of the third QW from the top. In such an SSA image, the deep blue color (white background) represents $\mathrm{GaN}$ and the red color (darkest region) corresponds to a $d$ value larger than 1.1 or indium content higher than $21 \%$. At the bottom of Fig. 4 , the color codes (grey levels) for various $d$-factor values are shown. In this image, one can see that the QW shape can still be identified although a cluster exists and indium atoms out-diffuse into the upper barrier. The horizontal and vertical line-scan profiles show that the local indium composition inside the cluster can be as large as $28 \%$.

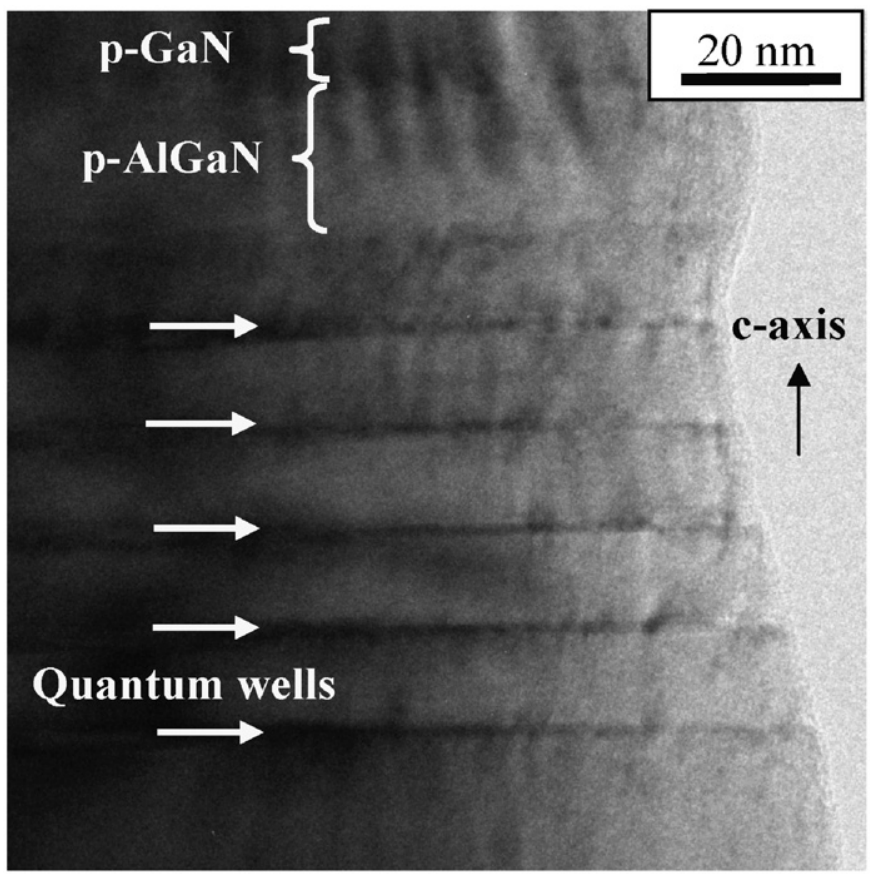

Fig. 2. A TEM image showing the five QWs of sample A.

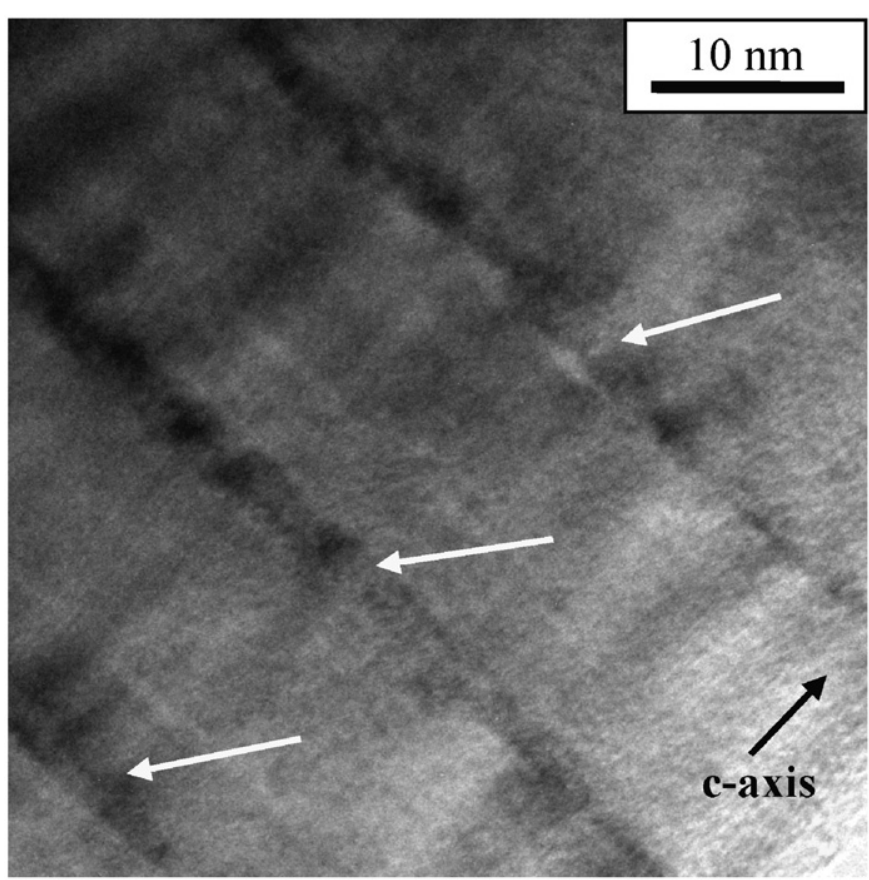

Fig. 3. A TEM image showing the nanostructures of the first three QWs (from the top) of sample A.

Fig. 5 shows a TEM image demonstrating the six QWs of sample B, as indicated with the white arrows. One can clearly see the weaker contrast of the bottom QW of low indium content, when compared with the five high-indium QWs above. A close look at the five high-indium QWs leads to the observation that the indium out-diffusion in 


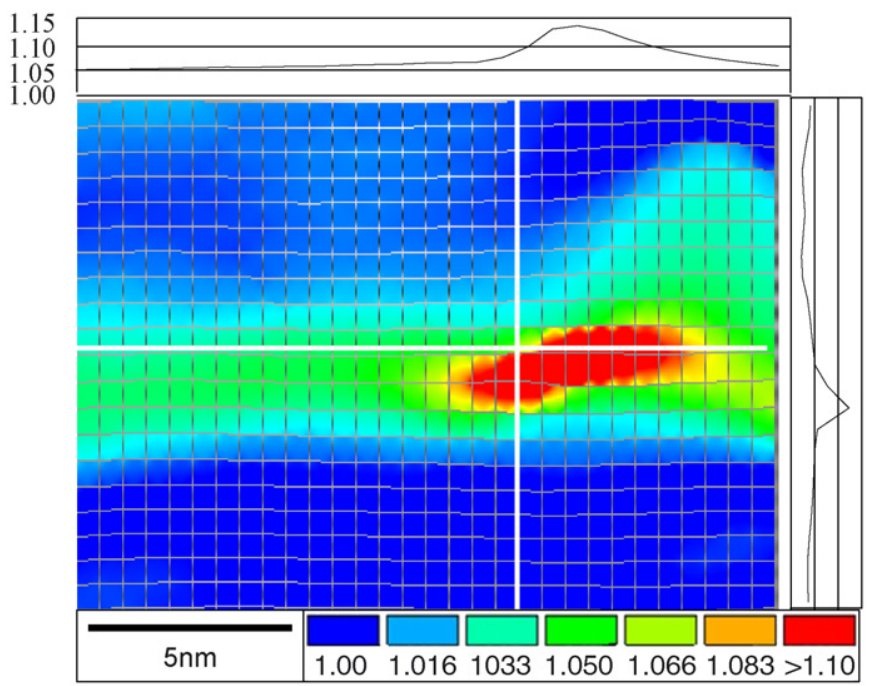

Fig. 4. A typical SSA image of a QW (the third QW from the top) of sample A. The numbers labeling the color coding represent the $d$ factor.

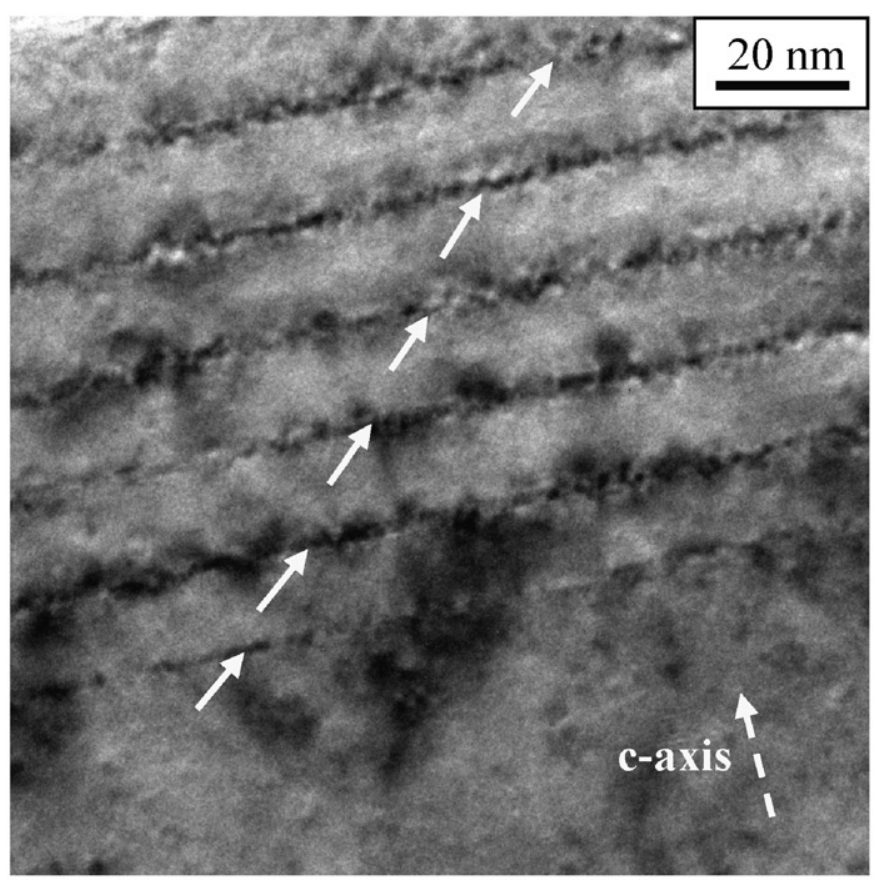

Fig. 5. A TEM image showing the six QWs of sample B.

those QWs closer to the low-indium one is stronger. The clustering behaviors in these QWs seem relatively stronger. Fig. 6 shows a TEM image demonstrating the nanostructures of the low-indium QW (the lower one in this image) and the first-grown high-indium QW. Because of the lowindium content, the lower dark stripe in the image looks lighter and more uniform, when compared with the other in the same image. The relatively lower indium content and more uniform indium distributions in this QW can also be seen in the SSA image in Fig. 7(a). Here, an almost uniform indium composition distribution can be clearly observed in

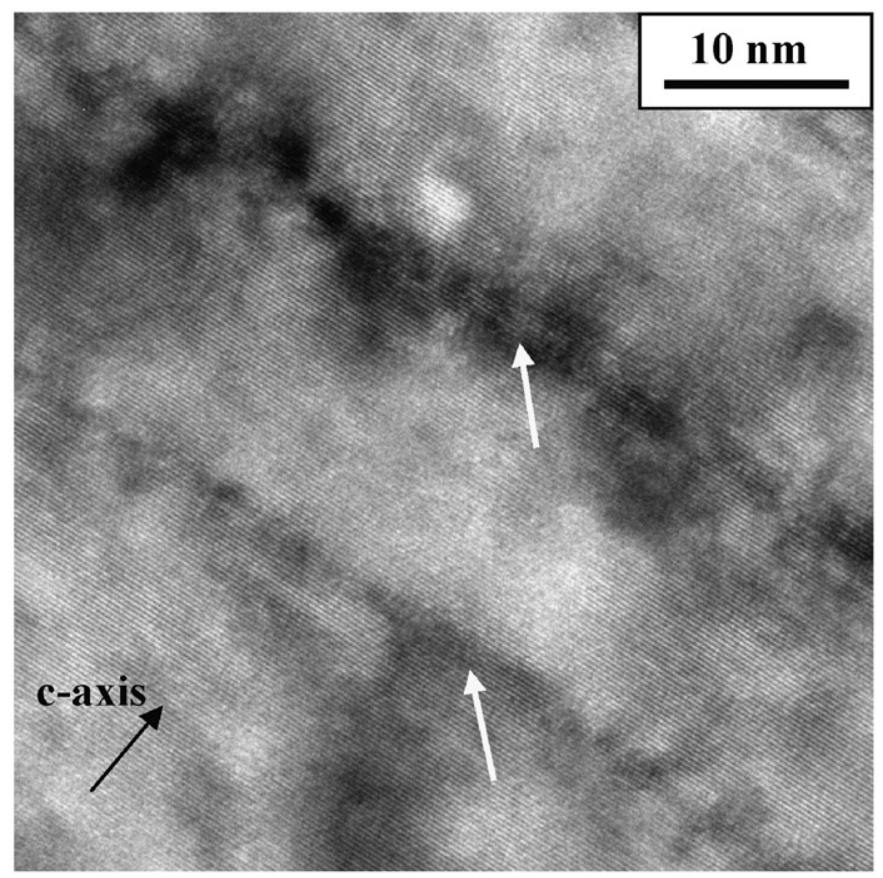

Fig. 6. A TEM image showing the nanostructures of a high-indium QW (the fifth from the top) and the low-indium QW (the lower one in this image) of sample B.

the horizontal line scan. In contrast, the SSA image of the first-grown high-indium QW demonstrates the highly irregular structure and strong clustering behavior (see Fig. 7(b)). As indicated with the line-scan results, the local indium content can be as high as $31.5 \%$ or even higher. In particular, the composition fluctuation range (crest-tovalley) is more than $21 \%$. For comparison, we show the SSA image of the top QW of sample B (see Fig. 7(c)). Here, the shape of QW is relatively better-defined. Nevertheless, the high-contrast cluster structures also exist.

To further understand the effect of the low-indium QW on the high-indium ones, we evaluate the average indium content of each QW in samples A and B by integrating the indium composition distribution in an SSA image and dividing by the designated QW area. We assume that all the indium atoms distributed around a QW in an SSA image belong to the QW. The results of the average indium contents of those QWs are listed in Table 1. In this table, indium contents are listed for those QWs counted from the top. As explained in the last section, the indium content of the low-indium QW in sample B is designated to be $7 \%$ based on the previous study. In this table, one can see that the indium contents among the five QWs in sample A are about the same. They slightly vary from $12.62 \%$ through $14.25 \%$ with the generally decreasing trend along the growth sequence. On the other hand, the five high-indium QWs in sample B show quite a different indium content distribution. From the top, the indium content increases from $14.62 \%$ with the QW depth and reaches the highest of $23.48 \%$ in the QW closest to the low-indium one. 


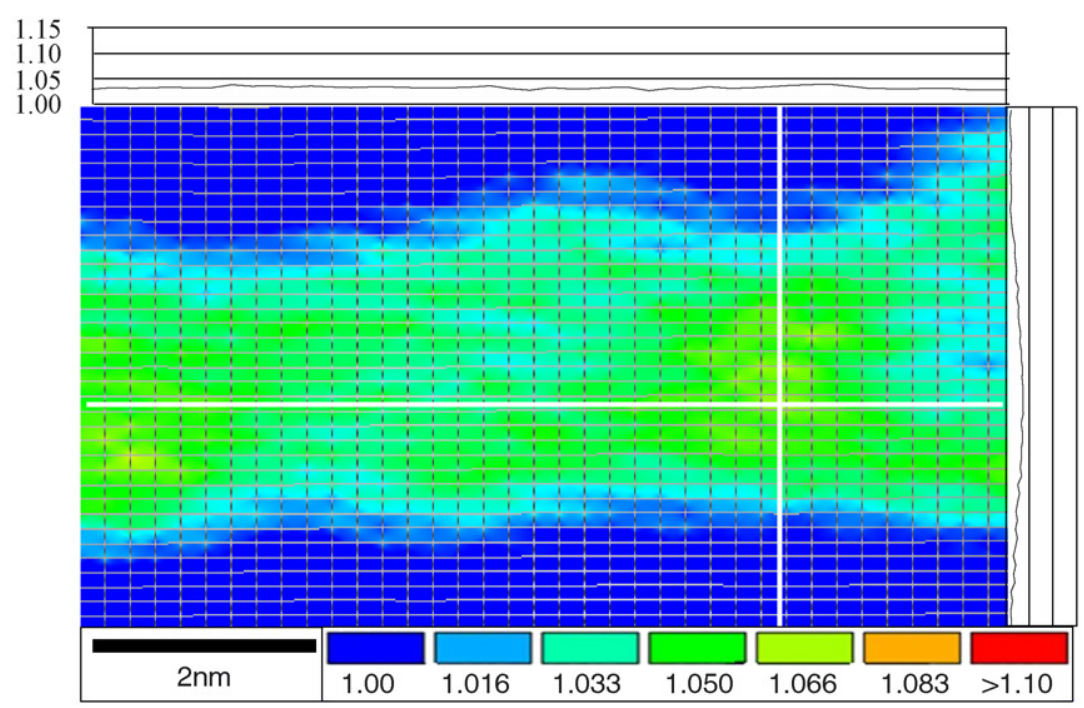

(a)

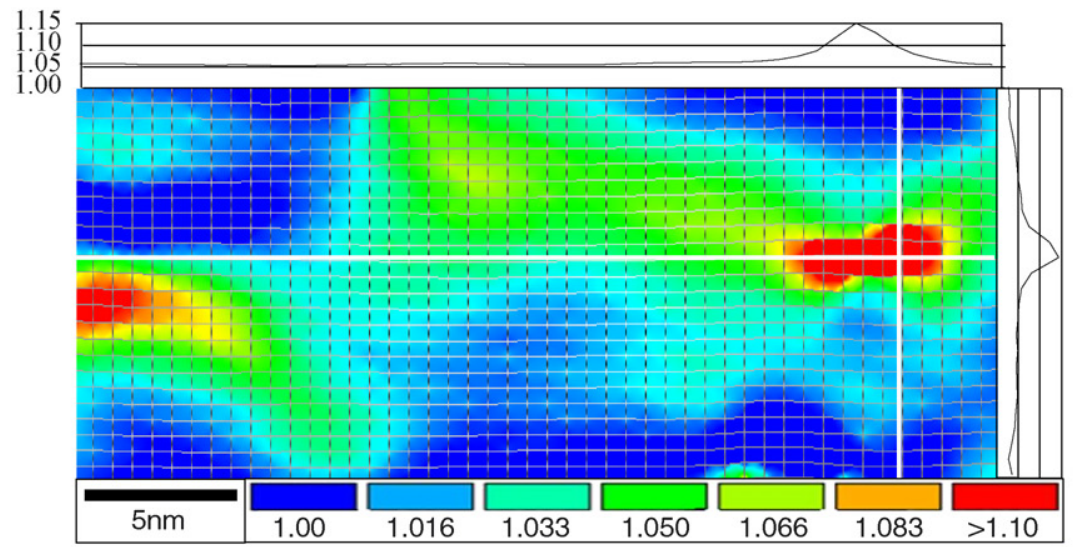

(b)

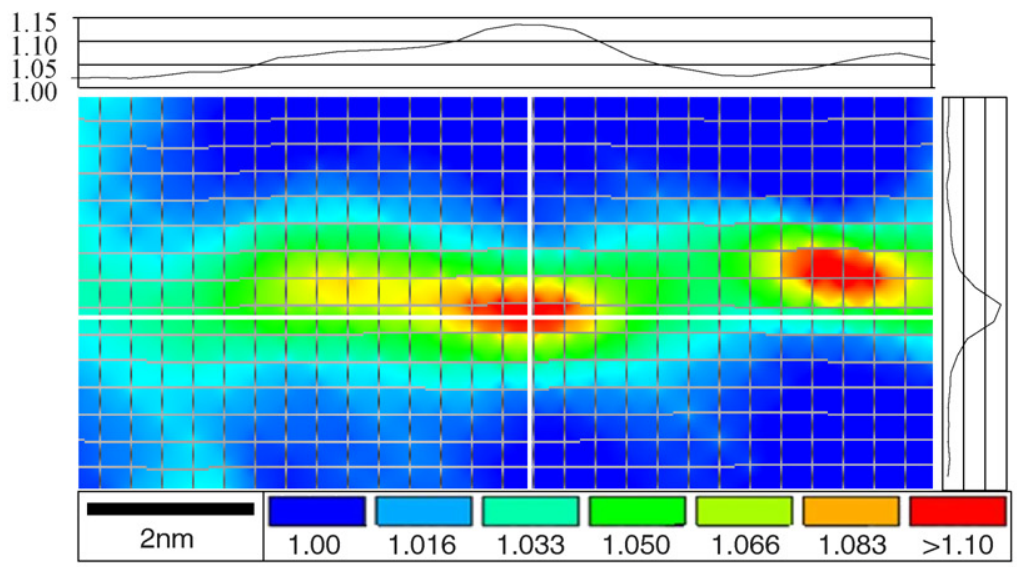

(c)

Fig. 7. Three SSA images of the QWs in sample B: (a) the low-indium (the sixth from the top) QW; (b) the first-grown high-indium QW (the fifth from the top); (c) the fifth-grown high-indium QW (the first from the top).

\section{Optical characterizations}

Figs. 8 and 9 show the temperature-dependent PL spectra of samples A and B, respectively. In sample A, the PL spectral peak stays around $2.3 \mathrm{eV}(539 \mathrm{~nm})$ in the whole temperature range. In sample B, two major peaks, with the stronger one at around $3 \mathrm{eV}(413 \mathrm{~nm})$ and the weaker one at around $2.18 \mathrm{eV}(569 \mathrm{~nm})$, can be observed. Because in the PL measurement the sample was excited from the top surface, the stronger PL emission around $3 \mathrm{eV}$ 
Table 1

Calibrated average indium contents of the QWs in samples A and B

\begin{tabular}{lll}
\hline & Sample A $(\%)$ & Sample B $(\%)$ \\
\hline QW1 & 12.62 & 14.62 \\
QW2 & 13.92 & 16.73 \\
QW3 & 12.90 & 20.31 \\
QW4 & 14.18 & 22.06 \\
QW5 & 14.25 & 23.48 \\
QW6 & & 7 \\
\hline
\end{tabular}

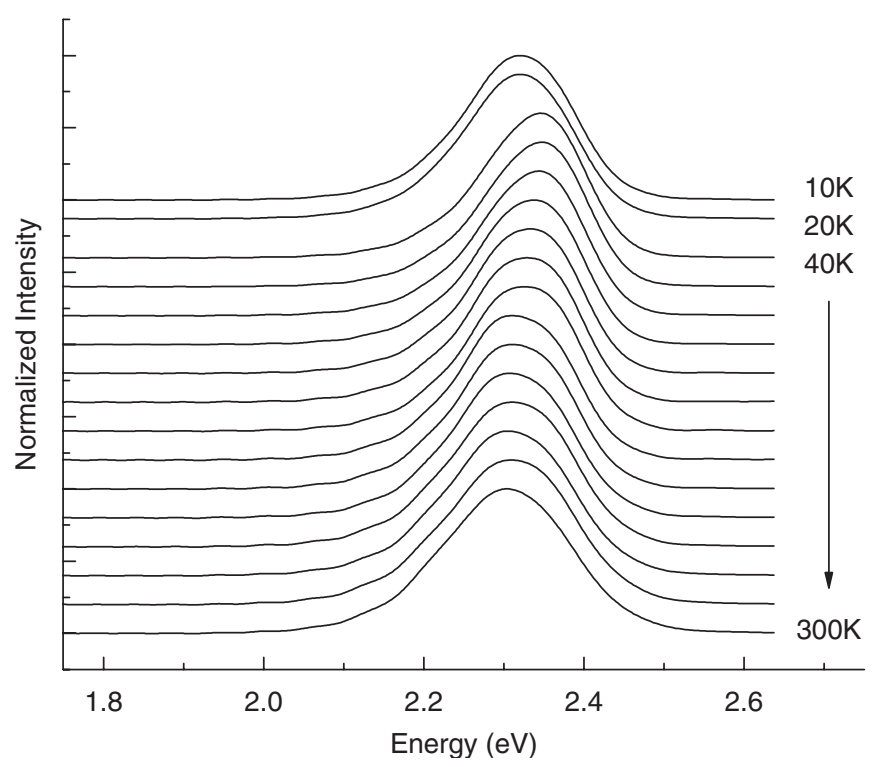

Fig. 8. Temperature-dependent PL spectra of sample A.

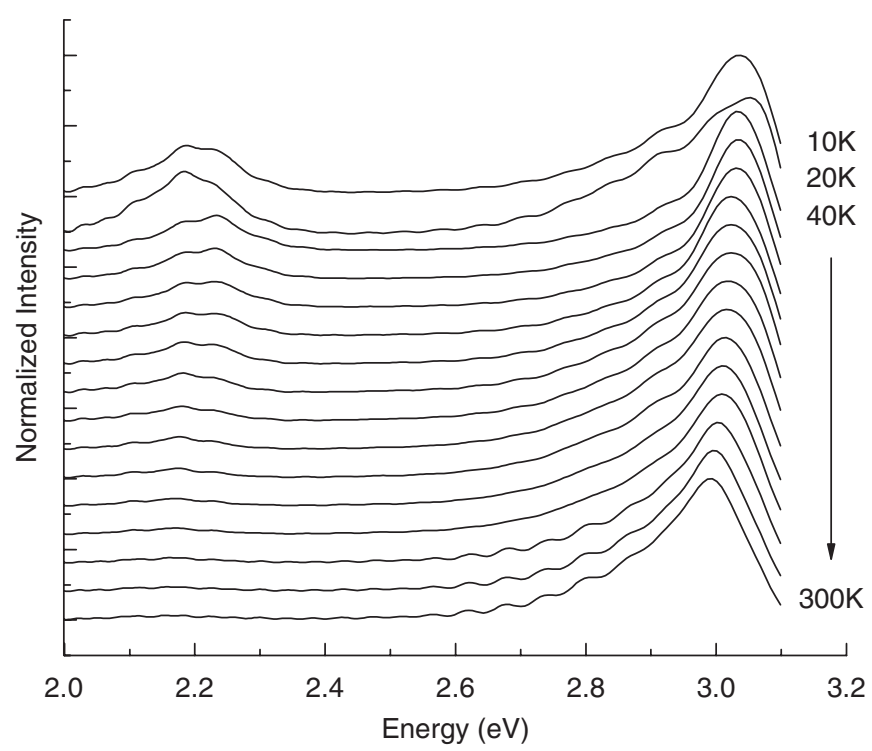

Fig. 9. Temperature-dependent PL spectra of sample B.

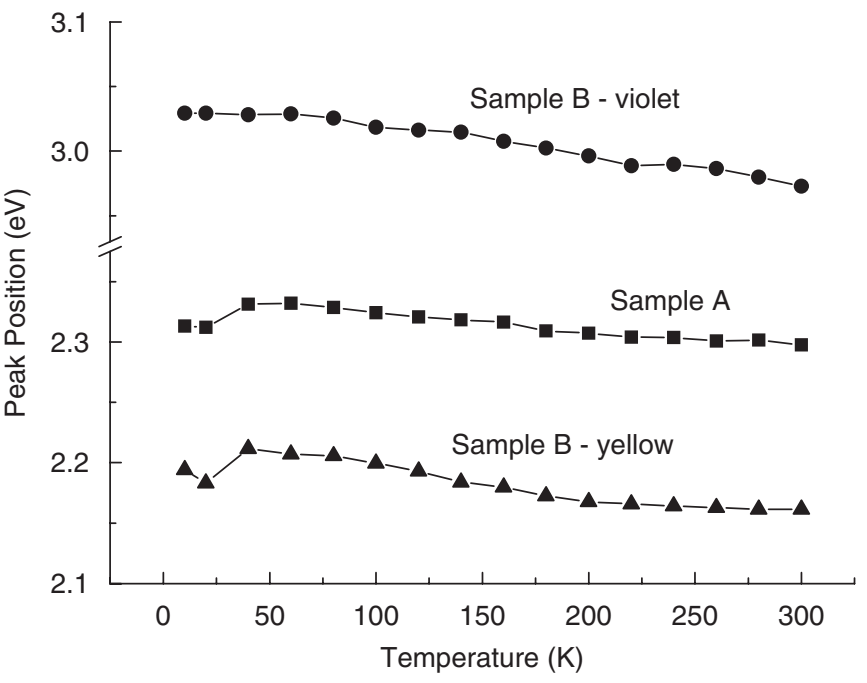

Fig. 10. Temperature-dependent PL spectral peak positions of the three peaks in samples A and B.

implies that the low-indium QW has higher crystal quality than the five high-indium ones in sample B. It is interesting to note that the high-indium QWs in sample B emit light of longer wavelength (around $569 \mathrm{~nm}$ ), when compared with that of sample A (539 nm), although the two sets of QWs were deposited under the same growth conditions. Fig. 10 shows the variations of PL spectral peaks with temperatures of the three emission features in Figs. 8 and 9. One can see the generally monotonically decreasing trend of the PL peak around $3 \mathrm{eV}$. However, the curves for the emissions of the two sets of high-indium QWs show the general trend of the S-shape variation. The S-shape variation is manifested with the trend of red-shift saturation beyond $200 \mathrm{~K}$ in either curve. Such an S-shape variation in temperature-dependent PL spectral peak position is usually attributed to the clustering behavior and/or quantum-confined Stark effect in an InGaN/GaN quantum well of high indium content [15-18].

Fig. 11 shows the thermal quenching effects of the three PL peaks in the two samples by plotting the normalized integrated PL intensities of the three peaks as functions of temperature. Because the curves of filled circles and filled triangles originate from the same spectral recording in the PL measurement, their relative level in this figure truly reflects the relative emission intensity. The weak temperature-dependent variation of the emission intensity from the low-indium QW of sample B confirms the relatively higher crystal quality. The almost parallel curves of the two sets of high-indium QWs imply their similar crystal quality although the set in sample B is slightly poorer.

\section{Discussions}

The higher indium content in the QW next to the lowindium one of sample $\mathrm{B}$, when compared with those in sample $\mathrm{A}$, is attributed to the enhancement of indium 


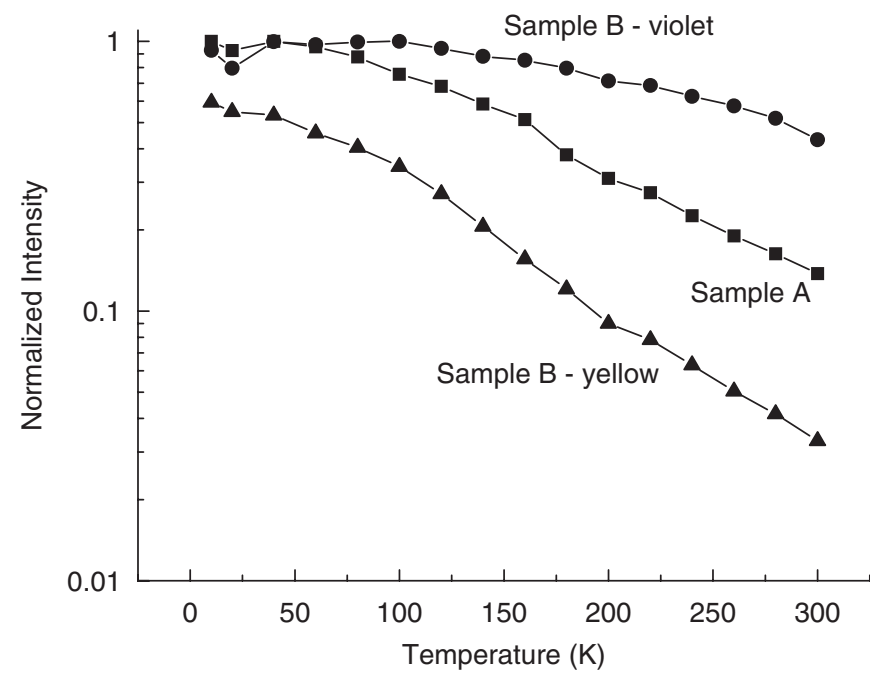

Fig. 11. Temperature-dependent integrated PL intensities of the three spectral peaks in samples A and B.

incorporation during the growth of this QW. According to the calibrated indium contents of those QWs (see Table 1), a QW closer to the low-indium one has a higher indium content, which leads to the emission of a longer wavelength. This trend of decreasing emission wavelength along the growth sequence of the high-indium QWs in sample B has been demonstrated in the depth-dependent cathodoluminescence measurement [19]. The depth-dependent cathodo-luminescence spectra show two peaks, with the short-wavelength peak intensity increasing and the longwavelength peak red-shifting with increasing electron penetration depth. The increased indium incorporation of the QWs above the low-indium one is due to its pre-strain effect.

Because of the large lattice mismatch between $\mathrm{GaN}$ and $\mathrm{InN}$, in an InGaN/GaN QW structure, influenced by the $\mathrm{GaN}$ barrier layer, the well layer normally experiences compressive strain. If such a strain is well preserved, the indium composition in the well layer is quite uniform. However, such a hetero-structure-induced strain can be partially released through the process of spinodal decomposition and hence the formations of composition fluctuations and indium-rich nano-clusters [4]. Such a strain relaxation process can occur particularly when the indium content is high (above several percents). When the heterostructure-induced strain is highly preserved, like the case of the low-indium QW in sample B, a barrier layer experiences certain tensile strain. This tensile strain increases with the indium content in the well layer until the aforementioned strain relaxation process occurs. Therefore, it is speculated that the low-indium QW in sample B is in the right condition of strain preservation for producing a significant tensile strain in the barrier layer right above it. The strain preservation condition is confirmed with the observation of uniform indium composition in this $\mathrm{QW}$, as shown in Fig. 7(a). With the significant tensile strain in the barrier layer, it is relatively easier for lattice matching and hence indium incorporation such that the average indium content in the well layer can be increased. Therefore, an average indium content as high as $23.48 \%$ in the first-grown high-indium QW can be achieved. Such a condition of pre-strained barrier can extend to the subsequently grown QWs. However, the effect diminishes with the number of QW. Therefore, the indium content decreases with the QW growth sequence. It eventually reaches the condition of sample A. On the other hand, in sample A, because the average indium content in each QW is so high that the strain relaxation process occurs and hence the tensile strain in the barrier becomes insignificant. Hence, the pre-strain effect does not exist in sample A. Therefore, the indium contents of the five QWs in sample A are about the same. The slight differences in the indium content among the five QWs in sample $\mathrm{A}$ are supposed to be due to experimental inaccuracies.

In growing high-indium InGaN/GaN QWs for implementing yellow-red emission, usually we need to reduce the growth temperature for increasing indium incorporation. However, with the decrease of the growth temperature, the crystal quality becomes poorer. To increase the indium incorporation without lowering the growth temperature, strain management is a key issue. The pre-strain effect generated by growing a low-indium QW of appropriate indium content is an effective approach for this purpose. In our implementation, the PL spectral peak has been shifted from 539 to $569 \mathrm{~nm}$. In a related research, we have also demonstrated that the electro-luminescence spectral peak is red-shifted from the green color of sample A into the orange-red color of sample B [20]. However, with the increased indium contents in those QWs, the crystal quality is slightly reduced, leading to the stronger thermal quenching effect between the two long-wavelength emission features in the two samples, as already shown in Fig. 11. This is an issue to be further investigated.

\section{Conclusions}

In summary, we have used the techniques of TEM and SSA to show the material nanostructures of two InGaN/ GaN QW samples. In one of the samples (sample B), a lowindium InGaN/GaN QW was grown before five highindium ones, which were grown under the same conditions as those for growing the five QWs in another sample (sample A). From the calibrations of the average indium contents of those QWs based on the SSA images, it was found that the QWs close to the low-indium one in sample $\mathrm{B}$ had higher indium contents than those in sample A. Such an increase of indium incorporation was attributed to the pre-strain effect of the low-indium QW on the barrier layer right above it. The pre-strain effect diminished along the growth of more QWs.

\section{Acknowledgements}

This research was supported by National Science Council, The Republic of China, under the Grant no. 
NSC 94-2120-M-002-006 and NSC 94-2115-E-002-015, and by US Air Force under the contract AOARD05-4085. Also, the support from the Center of Nano Science and Technology, National Taiwan University, is acknowledged.

\section{References}

[1] Y.S. Lin, K.J. Ma, C. Hsu, S.W. Feng, Y.C. Cheng, C.C. Liao, C.C. Yang, C.C. Chuo, C.M. Lee, J.I. Chyi, Appl. Phys. Lett. 77 (2000) 2988.

[2] Y.S. Lin, K.J. Ma, C. Hsu, Y.Y. Chung, C.W. Liu, S.W. Feng, Y.C. Cheng, M.H. Mao, C.C. Yang, H.W. Chuang, C.T. Kuo, J.S. Tsang, T.E. Weirich, Appl. Phys. Lett. 80 (2002) 2571.

[3] M. Rao, D. Kim, S. Mahajan, Appl. Phys. Lett. 85 (2004) 1961.

[4] I.H. Ho, G.B. Stringfellow, Appl. Phys. Lett. 69 (1996) 2701.

[5] E. Berkowicz, D. Gershoni, G. Bahir, E. Lakin, D. Shilo, E. Zolotoyabko, A.C. Abare, S.P. Denbaars, L.A. Coldren, Phys. Rev. B 61 (2000) 10994.

[6] T. Akasaka, H. Gotoh, T. Saito, T. Makimoto, Appl. Phys. Lett. 85 (2004) 3089

[7] T. Akasaka, H. Gotoh, H. Nakano, T. Makimoto, Appl. Phys. Lett. 86 (2005) 191920.

[8] M. Yamada, Y. Narukawa, T. Mukai, Jpn. J. Appl. Phys. 41 (2002) L246.
[9] D. Xiao, K.W. Kim, S.M. Bedair, J.M. Zavada, Appl. Phys. Lett. 84 (2004) 672.

[10] I. Ozden, E. Makarona, A.V. Nurmikko, T. Takeuchi, M. Krames, Appl. Phys. Lett. 79 (2001) 2532.

[11] Y.D. Qi, H. Liang, W. Tang, Z.D. Lu, K.M. Lau, J. Crystal Growth 272 (2004) 333.

[12] S.M. Ting, J.C. Ramer, D.I. Florescu, V.N. Merai, B.E. Albert, A. Parekh, D.S. Lee, D.V. Christini, L. Liu, E.A. Armour, J. Appl. Phys. 94 (2003) 1461.

[13] D. Gerthsen, B. Neubauer, A. Rosenauer, T. Stephan, H. Kalt, O. Schon, M. Heuken, Appl. Phys. Lett. 69 (1996) 2701.

[14] A. Rosenauer, U. Fischer, D. Gerthsen, A. Forster, Appl. Phys. Lett. 71 (1997) 3868.

[15] Y.H. Cho, G.H. Gainer, A.J. Fischer, J.J. Song, S. Keller, U.K. Mishra, S.P. DenBarrs, Appl. Phys. Lett. 73 (1998) 1370.

[16] T. Matsuoka, H. Okamoto, M. Nakao, H. Harima, E. Kurimoto, Appl. Phys. Lett. 81 (2002) 1246.

[17] S.F. Chichibu, A.C. Abare, M.S. Minsky, S. Keller, S.B. Fleischer, J.E. Bowers, E. Hu, U.K. Mishra, L.A. Coldren, S.P. DenBaars, T. Sota, Appl. Phys. Lett. 73 (1998) 2006.

[18] E. Berkowicz, D. Gershoni, G. Bahir, E. Lakin, D. Shilo, E. Zolotoyabko, A.C. Abare, S.P. Denbaars, L.A. Coldren, Phys. Rev. B 61 (2000) 10994.

[19] C.F. Huang, T.Y. Tang, J.J. Huang, W.Y. Shiao, C.C. Yang, C.W. Hsu, L.C. Chen, Appl. Phys. Lett., to be published

[20] H.S. Chen, C.F. Lu, D.M. Yeh, C.F. Huang, J.J. Huang, C.C. Yang, IEEE Photon. Technol. Lett., to be published 